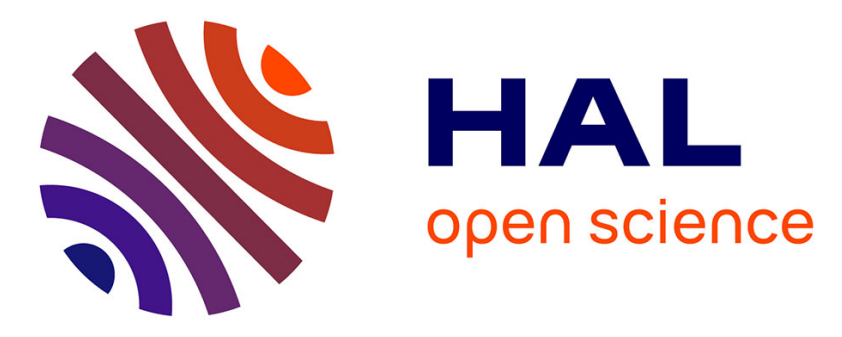

\title{
Analog Ultra Low-Power Acoustic Wake-Up System Based on Frequency Detection
}

Manon Fourniol, Valentin Gies, Valentin Barchasz, Edith Kussener, Herve Barthelemy, Remy Vauche, Hervé Glotin

\section{- To cite this version:}

Manon Fourniol, Valentin Gies, Valentin Barchasz, Edith Kussener, Herve Barthelemy, et al.. Analog Ultra Low-Power Acoustic Wake-Up System Based on Frequency Detection. 2018 IEEE International Conference on Internet of Things and Intelligence System (IOTAIS), Nov 2018, Bali, Indonesia. pp.109-115, 10.1109/IOTAIS.2018.8600849 . hal-02022330v2

\section{HAL Id: hal-02022330 \\ https://hal.science/hal-02022330v2}

Submitted on 6 Jan 2022

HAL is a multi-disciplinary open access archive for the deposit and dissemination of scientific research documents, whether they are published or not. The documents may come from teaching and research institutions in France or abroad, or from public or private research centers.
L'archive ouverte pluridisciplinaire HAL, est destinée au dépôt et à la diffusion de documents scientifiques de niveau recherche, publiés ou non, émanant des établissements d'enseignement et de recherche français ou étrangers, des laboratoires publics ou privés. 


\title{
Analog Ultra Low-Power Acoustic Wake-Up System Based on Frequency Detection
}

\author{
Manon Fourniol ${ }^{\dagger \ddagger}$, Valentin Gies ${ }^{\dagger \ddagger}$, Valentin Barchasz $z^{\ddagger}$, Edith Kussener ${ }^{\dagger}$, \\ Hervé Barthelemy ${ }^{\dagger}$, Remy Vauché ${ }^{\dagger}$ and Hervé Glotin ${ }^{\S}$ \\ ${ }^{\dagger}$ Aix Marseille Université, Université de Toulon, ISEN, CNRS, IM2NP - UMR 7334, Marseille, France \\ ${ }_{\S}^{\S}$ Aix Marseille Université, Université de Toulon, CNRS, LIS, DYNI Team, Marseille, France \\ ‡Université de Toulon, SMIoT, Toulon, France
}

\begin{abstract}
An ultra low power acoustic wake-up detector based on high frequency signal analysis is presented in this paper. Focused on environmental or military Internet of Things (IoT) applications, it aims at detecting in real time the presence of specific animal species or drones for generating alerts and for triggering power consuming tasks such as high frequency signal recording only when needed.

This wake-up detector continuously monitors the presence of specific frequencies in an analog acoustic signal, with a good frequency selectivity and a high frequency detection capability. It is based on an ultra-low analog frequency to voltage converter using a current-mirror, analog timers and comparators.

Dedicated to long term stealth environmental or military surveys, a strong emphasis has been put on power consumption reduction in order to limit size and weight of the system. This power consumption has been reduced to $34 \mu \mathrm{W}$, leading to a full year of autonomy including the microphone when powered by 3 coin cell CR2032 batteries.
\end{abstract}

\section{INTRODUCTION}

Internet of Things (IoT) systems for environmental and military surveys are submitted to strong operational or environmental constraints, such as installing a device in a strict nature reserve or in a restricted area. Environmental and military surveys requires to record signals such as sound for long periods of time (several months or years). These recording operations are hardly compatible with low power and high frequency recording because of data storage capacity or battery energy.

For example, acoustic recording of animals such as bats or rats, having a communication and localization system emitting up to $120 \mathrm{kHz}$ [1] and aerial drones producing a characteristic noise around $40 \mathrm{kHz}$ [2], requires to have a data acquisition system working at a minimum of $240 \mathrm{ksps}$ with a resolution of 24 bits for low level signal detection. This leads to store $2.5 G B$ of data every hour for each channel.

In these conditions, it is almost impossible to have a portable autonomous long term data acquisition system able to record continuously. We have to cope with an issue described in [3][5]. In order to store efficiently the high frequency acoustic signals occurring sparsely at a random time, it is more efficient to start recording only when an interesting signal is present. This allows to avoid useless power consumption, useless data storage, and useless post-processing for rising alarms or finding out interesting events. Following the emerging idea of using edge computing techniques, this local prefiltering process allows to detect only interesting events, thus consuming less, saving a huge amount of data storage and making the recorder operational for a longer time.

Existing state of art environmental recording systems [6] [7] are working on a standard $12 \mathrm{~V}-1.2 \mathrm{Ah}$ lead battery having a global capacity of $14.4 \mathrm{Wh}$. In continuous high frequency recording, batteries would last only 30 hours, without including the energy necessary generating alerts. Fortunately, continuous recording is mostly not necessary for environmental rare event detection, but can be reduced to interesting data periods, for example for detecting intrusions of specific species in a strict natural reserve or malicious behaviors such as aerial drones invasions in a restricted military area.

A solution allowing long term monitoring and alert generation is to use a wake-up detector activated by detection of a specific frequency on the acoustic signal, and to record the signal only when the wake-up detector has been triggered. Low power wake-up systems based on raw frequency detection exist [8]-[11] but they are mainly specified on detecting a specific low frequencies [12], [13] or are specialized in radio frequency analysis, for example RFID technologies [14]. There are also some detectors using advanced digital processing techniques and having more developed capabilities such as pattern detection, like [15] or [16], [17], but with a limited sampling frequency or at a higher overall power cost due to analog to digital conversions.

In [18], we have introduced a way for detecting environmental events using a digital wake-up detector working on high frequency sound signal has been proposed. Power consumption of this system is about $0.6 \mathrm{~mW}$. That is equivalent to the power consumption of the ultrasonic microphones used (Knowles SPU0410LR5H). Using a $14.5 \mathrm{Wh}$ battery (classical $12 \mathrm{~V}-1.2 \mathrm{Ah}$ lead battery), this micro-controller based wake-up system can be active about 3 years in continuous mode, compared with a 30 hours of continuous high frequency recording with most of the time no useful signal. This is paving the way for long term environmental survey protocols, but with a major drawback : it is impossible to use it in very lightweight and small embedded systems due to the lead battery size which is $10 \mathrm{~cm}$ long and weights $600 \mathrm{~g}$. 
In this paper, we present a novel implementation of a high frequency signal wake-up detector based on an analog implementation using discrete components as shown in Fig. 1.

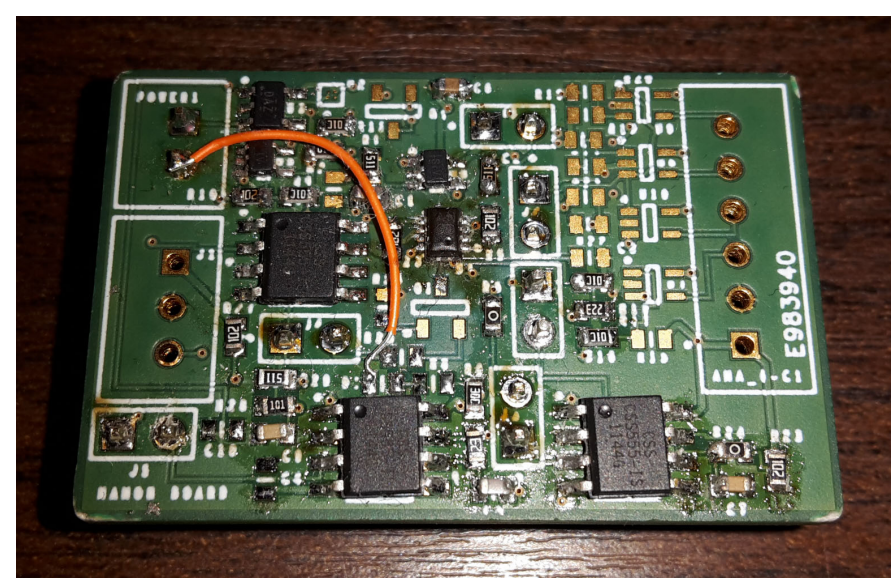

Fig. 1. Ultra-low power analog wake-up detector

First, the general architecture of the acquisition system is presented with a focus on the power consumption budget available for waking-up on interesting signal detection, having this detector work in always on mode. We call it the wake-up always on mode. In a second part, the ultra-low power analog implementation of the wake-up system based on frequency analysis is described. In a third part, power consumption results are presented and compared to existing solutions.

\section{Acoustic Environmental Monitoring System DESCRIPTION}

\section{A. Size and weight reduction : a strong constraint on batteries}

As explained in the introduction, compared to previous solutions, we aim at reducing the size and the weight of the data acquisition system to embed it anywhere without disturbing present species.

We mainly focus on the battery size reduction by using 3 standard $3 V-0.23 A h-0.69 W h$ CR2032 batteries (weighting $3 \mathrm{~g}$ each) instead of a classical $12 \mathrm{~V}-1.2 \mathrm{Ah}-14.4 \mathrm{Wh}$ lead battery (weighting 600g) as presented in [18]. Battery weight has to be reduced by a factor 100 whereas capacity has to be reduced by a factor 10 . At the same time, lifetime of the detection system must be maintained in wake-up always on mode for at least one year. That means power consumption has to be reduced by approximately a factor 10 to compensate the battery reduction.

Considering that alerts to be detected and recorded are rare enough to be neglected, we can assume that the maximum acceptable average power consumption in wake-up detection mode is approximately equal to the battery capacity divided by the needed autonomy of the system (i.e. 12 months). It is the same for aerial drones detection systems, considering intrusions are rare. For example, using 3 coin cell batteries CR2032, this leads to allow an average power budget on one year of $\frac{3 \times 0.69}{24 \times 365}=236 \mu \mathrm{W}$ for the always on wake-up detection mode including the microphone power supply. With this constraint, use of the Knowles SPU0410LR5H microphone used in [18] is not possible because its power consumption is $500 \mu \mathrm{W}$. Instead, we have chosen another lower power microphone from Knowles, the $F G-23329-P 07$ having a power consumption of about $200 \mu \mathrm{W}$. This microphone has been validated experimentally for ultrasonic detection at $40 \mathrm{kHz}$ or more without any trouble, even if it is not described in the corresponding Knowles datasheet. With this microphone, $36 \mu \mathrm{A}$ are available for powering the wake-up always on detector.

\section{B. System architecture}

This paper focuses on the low power wake-up system based on frequency analysis, however a global description of the recording and analysis system is presented in Fig. 2. The acoustic low power wake-up system based on frequency analysis is connected with other parts of the high frequency recording system using a low power device. Processing is done in three steps, each one having an increasing power cost :

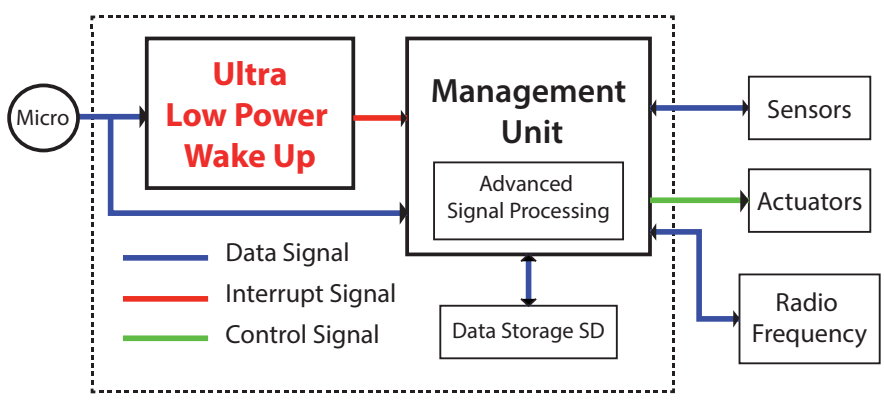

Fig. 2. Global recording system description.

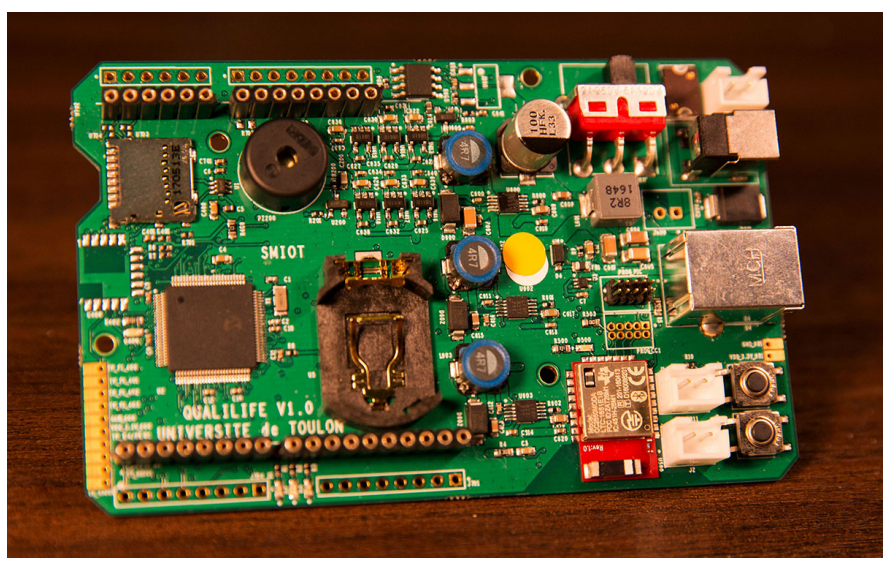

Fig. 3. Low Power Acoustic Wake-Up System integrated in "Qualilife" recording system

1) First, a microphone captures the acoustic signal and transmits it to our low power analog wake-up detector based on frequency analysis. When the wake-up detects 
a specific frequency sound distinctive of an animal, an interrupt is sent to the management system.

2) Upon reception of an interrupt, the management unit microcontroller starts further advanced signal processing using traditional linear or non-linear filters, neural networks, wavelets transform or correlations to determine whether the signal detected is a true alert or not [19][23]. Other additional sensors can be used to improve the quality of these additional computations. These operations are performed on demand, with a more important power consumption of about $17.5 \mathrm{~mW}$. The management unit is also able to launch an alert. Theses filtering operations are not in the scope of this paper.

3) Then, if the detection is confirmed, the management unit starts recording the signal from the microphone and stores it on a SD card for a defined amount of time, in order to record the next occurrences of the interesting signal. It is important to notice that this strategy cannot be used for recording one shot signals because signal used for low power acoustic wake-up is lost. A solution using an analogical to digital signal buffer converter could be implemented but would not be low power.

\section{Ultra Low-Power WAKe-Up Detector BASED ON FREQUENCY ANALYSIS ANALOG IMPLEMENTATION}

The analog low power wake-up detector has been designed with an idea of simplicity in order to get the lowest power consumption possible. It is based on a frequency to voltage conversion, using a monostable driven linear discharge of a capacitor at a constant current. Periodically, the voltage of the capacitor, image of the input frequency, is sampled and hold for being compared to thresholds by analog comparators.

This implementation has been inspired by our previous digital implementation on a PIC24FJ256 [18], [24] from Microchip, but with a drastic reduction of power consumption.

\section{A. Architecture Description}

As shown on Fig. 4, signal from the analog low power microphone is first passed into an hysteresis comparator. This comparator allows ambient noise to be removed by triggering only when a the $U_{S}$ input signal have a sufficient amplitude.

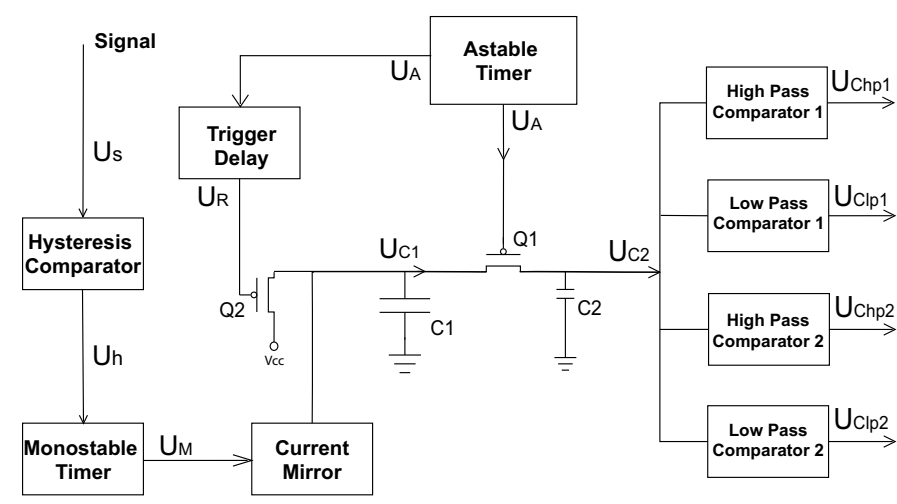

Fig. 4. Ultra Low Power Acoustic Wake-Up System
Then, output $U_{h}$ of the hysteresis comparator drives a monostable analog timer which will generate pulses $U_{M}$ with a constant high time, but synchronized with the input signal. At this step, we can notice that $U_{M}$ average value is directly proportional to the input signal $U_{S}$ frequency. At this step, a simple $R C$ filter could be used, but the frequency to voltage conversion would not be linear due to the exponential charges of the $R C$ filter. Instead of that, we need a perfectly linear conversion in order to determine accurately the input frequency.

This linear conversion is done using a current mirror coupled to a capacitor $C_{1}$. This has been inspired by our preceding implementation as shown in [18]. The current mirror is used like a Charge Time Measurement Unit (CTMU) [25] present in Microchip micro-controllers. Pulses from the monostable timer create a constant current in the left transistor of the current mirror. This current is mirrored to get $I_{C_{1}}$ which discharges the capacitor $C_{1}$. Thus, voltage $U_{C 1}$ decreases linearly during the monostable pulses, and remains constant during the other phases as shown in Fig. 5.

Equation 2 describes $U_{C 1}$ evolution, corresponding to waveforms presented at Fig. 5 :

$$
\begin{aligned}
U_{C 1} & =V_{C C}-\int_{0}^{t} \frac{I_{C 1}(t)}{C_{1}} d t=V_{C C}-\int_{0}^{t} \frac{I_{0}}{C_{1}} U_{m}(t) d t \\
& =V_{C C}-\frac{I_{0}}{C_{1}} * N * T_{P W} \\
& =V_{C C}-\frac{I_{0}}{C_{1}} * \frac{F_{S}}{F_{S H}} * T_{P W}
\end{aligned}
$$

where $U_{m}$ is a logical level 1 or 0 depending on the monostable output state, $I_{0}$ is the current during monostable pulses in the current mirror, $T_{P W}$ the pulses duration, $N$ the number of pulses between two successive resets, $F_{S H}$ the sample and hold frequency, and $F_{S}$ is the input signal frequency.
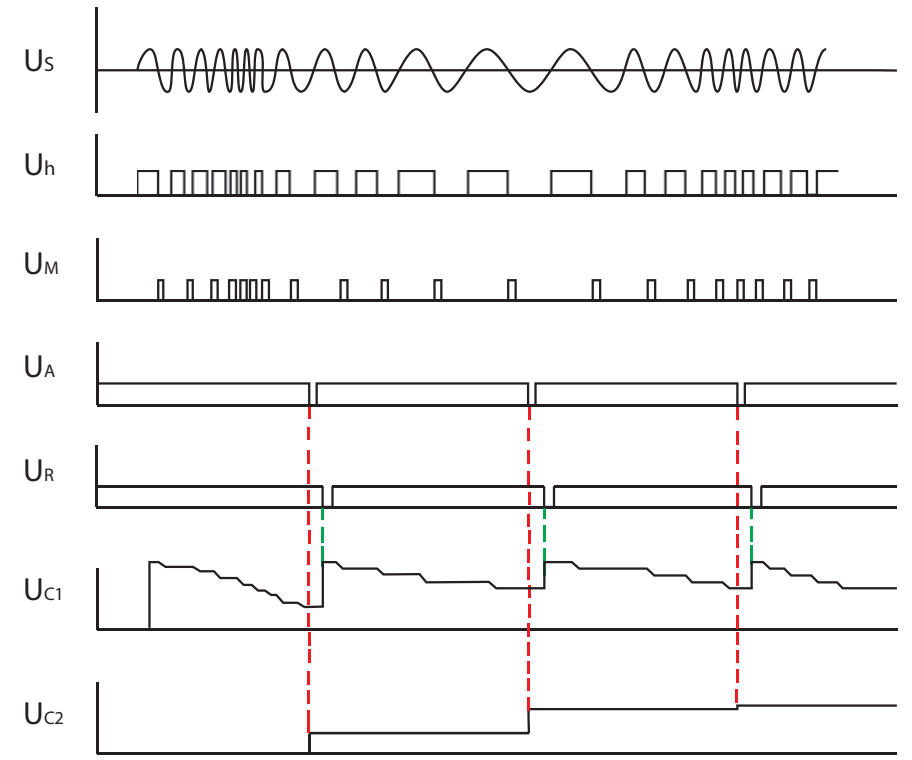

Fig. 5. Analog Wake-up detector waveforms 
$U_{C 1}$ voltage is an image of the frequency and have to be read periodically. This is done using a low frequency astable timer producing periodically a signal $U_{A}$ at a fixed frequency $F_{S H}$, for closing $Q_{1}$ PMOS transistor. $U_{C 1}$ voltage is sampled and hold into $U_{C 2}$, considering that $C_{1}$ capacitor value is much more important than $C_{2}$ capacitor value. While this sample and hold operation has been done, $Q_{1}$ transistor is opened and $Q_{2}$ PMOS transistor is closed in order the reset the voltage $U_{C 1}$ value at $V_{C C}$.

Consequently, $U_{C 2}$ voltage is an image of the input signal frequency. Final step of the process is to compare $U_{C_{2}}$ with fixed values in order to determine if the measured frequency is within or not a specified interval. An ADC could also be used to get the frequency value, but it would lead to a tremendous power consumption compared with the overall consumption of our wake-up trigger.

\section{B. Implementation}

Implementation of this ultra low-power wake-up detector has been done on a PCB board using ultra low power analog components. For example, $3.3 \mathrm{~V}$ reference voltage is done using a ultra low power regulator TPS783 [26] that regulates the input voltage to $3.3 \mathrm{~V}$ while consuming only $500 \mathrm{nA}$.

1) Input Comparators: The input signal $U_{S}$ is first processed by a hysteresis trigger as shown in Fig. 4. The hysteresis comparator outputs the $U_{h}$ signal. A small ambient noise will not trigger the hysteresis comparator, thus eliminating the input low level perturbations. Compared to the single comparators used in [18], the hysteresis comparator offers a more reliable noise rejection. In order to adapt filtering to the different detection sensitivity, hysteresis level can be adjusted by a reference voltage generated by the DAC of the supervisor.

Choice of the comparator is important because of the tradeoff between operating frequency and power consumption. TVL3691 [27] is a good candidate with its very low power consumption (only $75 n A$ ). However, its maximum switching frequency $(30 \mathrm{kHz})$ is too low for high frequency operations such as bats detection $(100 \mathrm{kHz})$. Another reference has been chosen : TLV7031 [28] from Texas Instrument having a propagation delay comprised between $3 \mu$ s and a supply current of $335 n \mathrm{~A}$. It allows to process signal up to $300 \mathrm{kHz}$.

2) Timers: Ultra low power timers are a key for our analog wake-up detector. They are used for monostable pulses generation synchronized with the rising edges of the input signal, for astable sample and hold signal $\left(U_{A}\right)$ generation and for generating a delayed signal $\left(U_{R}\right)$ resetting capacitor voltage after a sample and hold operation. This requires the use of three timers. Due to power constraints, a very low implementation of the classical 555 timer has been chosen : the CSS555C [29] consuming only $3 \mu A$ and covering all our needs [30].

- Monostable timer : signal $U_{h}$ from the input comparators triggers a monostable timer, producing a rising edge $U_{M}$ on each falling edge of the input signal. Fig. 6 shows the hardware implementation using CSS555. Pulse width $T_{P W}$ is equal to (equation 3 ) :

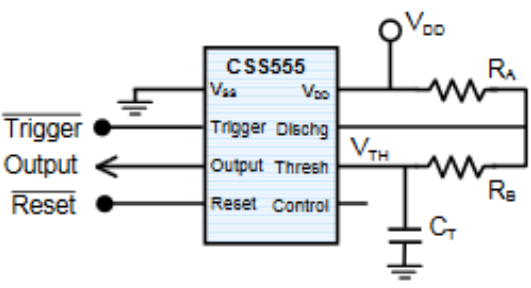

Fig. 6. Monostable Timer.

$$
T_{P W}=L\left(R_{A}+2 R_{B}\right) C_{T}
$$

where $L=0.695$. In order to process input signals at $100 \mathrm{kHz}, T_{P W}$ has been chosen equal to $0.66 \mu \mathrm{s}$, leading to $R_{A}=10 \Omega, R_{B}=0 \Omega$ and $C_{T}=100 \mathrm{pF}$. It is interesting to notice that lower capacitor values $C_{T}$ have been tested, but the pulse width was less precisely defined, due to parasitic capacitors not negligible under these values.

- Astable low frequency timer : Astable signal is also generated by a CSS555 timer producing a periodic signal $U_{A}$ as shown in Fig.7. Astable frequency is set using equation 4:

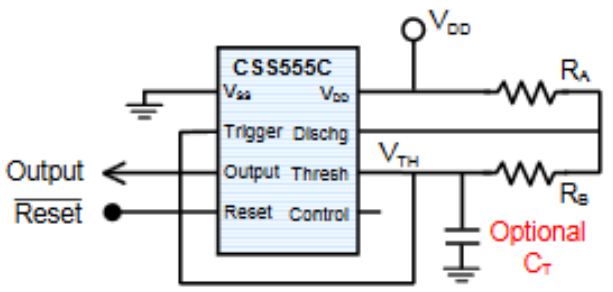

Fig. 7. Astable Timer.

$$
T_{O S C}=L\left(R_{A}+2 R_{B}\right) C_{T}
$$

where $L=0.695$. In order to obtain a sample and hold timer frequency of about $1 \mathrm{kHz}, R_{A}=700 \mathrm{k} \Omega$, $R_{B}=370 k \Omega$ and $C_{T}=1 n F$ have been chosen.

The capacitor reset signal $U_{R}$ is obtained for $U_{A}$ by generating a pulse with a delay using a CSS555 as shown in Fig.8. Delay $t_{D}$ and pulse width $t_{P W}$ is set according to equation 5 :

$$
\begin{gathered}
t_{D}=\frac{1}{2} C_{T}\left[t_{d 0}\left(R_{A} / / R_{F}+R_{B}\right)+\left(t_{d 1} R_{B}\right)\right] \\
t_{P W}=\frac{1}{2} C_{T} L\left(R_{A} / / R_{F}+2 R_{B}\right)
\end{gathered}
$$

where $L=0.695$ and $t_{d 0}=\ln \left(\frac{2}{3}\right)-\ln \left(1-\frac{2}{3}\left(R_{F}+R_{A}\right) / R_{F}\right)$.

In order to have a delay of $20 \mu \mathrm{s}$ allowing to correctly transfer charges form $C_{1}$ to $C_{2}$ before reset, following values 


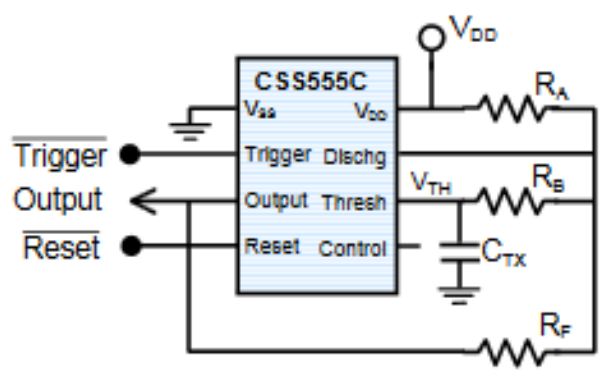

Fig. 8. Delay Timer.

have been chosen : $R_{A}=100 k \Omega, R_{B}=10 k \Omega, R_{F}=510 k \Omega$ and $C_{T}=100 \mathrm{pF}$.

3) Current Mirror: Current mirror is the last central element of our system. It is replacing the CTMU [25], [31], [32] used in [18]. The current mirror is used as a constant controlled current source driven by the monostable output $U_{M}$ for discharging main capacitor $C_{1}$. When activated, current value in the mirror $I_{0}$ is set by the serial resistor and the output voltage of the monostable as shown in equation 7 .

$$
I_{0}=\frac{V_{C C}-V_{B E}}{R}
$$

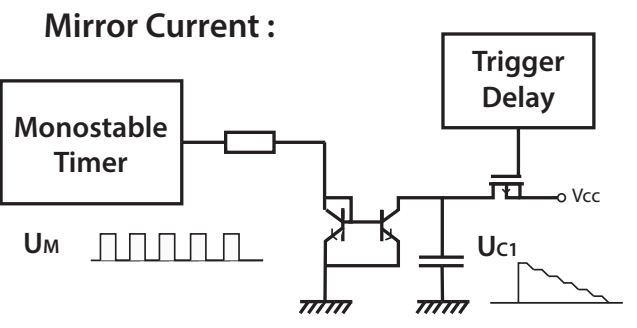

Fig. 9. Current Mirror

Choice of the current mirror analog component is very important as precision of frequency measurement relies directly on the mirror precision. BCV62 PNP current mirror [33] has been tested at a first time because of its low cost. But its precision was very low (70\% of matching between transistor $\mathrm{T} 1$ and transistor T2) and not suitable for a precise detection of frequency. Then, REF200 [34] from Texas Instrument has been chosen. This expensive current mirror is very precise, with around $0.25 \%$ of matching between the two transistors. It works up to $5 \mathrm{MHz}$ which is more than sufficient for our $100 \mathrm{kHz}$ signal. Power consumption is not a problem for the mirror considering it is only consuming power when monostable is triggered, that means rarely. The choice of the REF200 has an impact on the design. Using NPN transistors, capacitor $C_{1}$ is being discharge by the mirror, whereas a PNP mirror could be used for charging the capacitor.

4) Capacitors and associated currents: Capacitor $C 1$ is the heart of the analog wake-up detector, being charged linearly by successive phases. The values of $C_{1}, C_{2}$ and $I_{0}$ have to be chosen carefully. Considering input frequency to be detected is
$100 \mathrm{kHz}$, sample and hold frequency is $1 \mathrm{kHz}, T_{P W}=0.66 \mu \mathrm{s}$ and $U_{C_{1}}$ minimal value is 0 , equation 2 gives :

$$
\frac{I_{0}}{C_{1}}=\frac{V_{C C}}{\frac{F_{S}}{F_{S H}} * T_{P W}}=50000
$$

Limiting the mirror current to $I_{0}=10 \mu \mathrm{A}$ conducts to have $C_{1}=20 n F$.

After integration, $U_{C_{1}}$ voltage have to be sampled and hold in $C_{2}$. This is done by closing $Q_{1}$ PMOS. Charges are transferred from the $C_{1}$ to $C_{2}$, leading to a change in $U_{C_{1}}$. In order to keep this change a low as possible, $C_{2}$ value have to be chosen far lower than $C_{1}$ and has been set to $C_{2}=1 n F$.

5) Output Comparators: Once the $U_{C_{1}}$ has been sampled and hold into $C 2$ capacitor, $U_{C_{2}}$ has to be exploited in a very efficient and simple way. This is done using ultra low power and low speed comparators as shown on Fig.10.

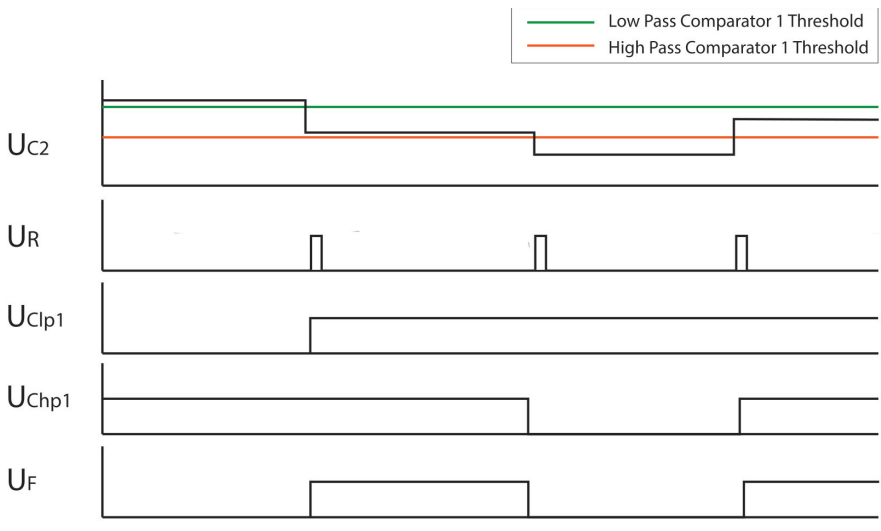

Fig. 10. Final Computation

By associating 2 comparators, a specific range of input frequency can be selected in output with a good precision. If the input signal frequency is in the selected interval, both outputs of the comparators will be equal to a logical 1, triggering an interrupt on the management unit.

Several comparators associations can be done, allowing to detect a signal in several bands of frequencies. This can be used for detecting generated frequencies in order to synchronize or trigger a wireless sensor network as presented in [35].

As the output comparators are working at a low frequency, is not necessary to use a fast comparator such as the TVL7031. Instead of that, the TVL3691 can be used, leading to an additional power reduction.

\section{RESULTS}

Our ultra-low power wake-up detector has the ability of detecting signals having a frequency up to $300 \mathrm{kHz}$. As we can see on Fig. 4, three comparators TLV7031 are present in the design, each one having a power consumption of $0.335 \mu \mathrm{A}$. Three analog functions using a CSS555C are also used, each one having a power consumption of about $3 \mu \mathrm{A}$ under $3.3 \mathrm{~V}$. Power consumption of the current mirror is very low, because this component is only active on the very short monostable pulses, with an active current limited to $10 \mu \mathrm{A}$. This is also true 
for the resistors around the CSS555C. Both MOSFETs are also used sparsely and thus have a very limited power consumption compared to the CSS555C. Adding all power consumptions, this leads to an approximate overall consumption of $36 \mu \mathrm{W}$ for the analog wake-up detector.

This power consumption can be reduced by replacing the TLV7031 by the TLV3691 which consume only $75 n A$ but is limited to $30 \mathrm{kHz}$. This replacement can be done only on the final comparators considering they are working at a low frequency (less than $1 k H z$ ). We this replacement, power consumption is reduced to $34 \mu \mathrm{W}$.

This power consumption can be compared with the microcontroller implementation of the acoustic Wake-up detector presented in [18]. Its power consumption is about $600 \mu \mathrm{W}$. That means our analog wake-up detector power consumption is 18 times lower than this previous implementation.

This allows the system to detect high frequency analog wake-up events in continuous mode during 2.3 years on a single CR2032 coin cell having a $230 \mathrm{mAh}$ capacity, without considering the microphone. Used with a low power microphone FG-23329-P07 from Knowles, the power consumption raises to $230 \mu \mathrm{W}$, leading to a continuous detection of 125 days using a single $C R 2032$ coin cell. A one year autonomy can be achieved using 3 coin cells, having a total weight of 9 grams. [36].

\section{CONCLUSION AND FUTURE WORKS}

The ultra-low power $(34 \mu \mathrm{W})$ always-on wake-up detector presented in this paper is lightweight and small. It can be used for applications in environmental or military surveys such as stealth intelligent recording of acoustic signals emitted by animals or drones. Capable of detecting high frequency acoustic signals in a very low power mode, it is used as a prefiltering stage for detecting instants when an input signal having a chosen frequency is present or suspected to be there.

This solution is perfectly suited for long term embedded stealth monitoring systems used in environment or military applications. This analog wake-up detector shown in Fig. 1 is used for protecting a strict nature reserve island (Ilot Bagaud, part of the Parc National de Port Cros in France) from rats intrusions, combined with the Qualilife system [7], [36] for recording, analyzing and transmitting alerts in real time.

Other applications using non acoustic high frequency signal analysis and detection can be developed using our analog Wake-up detector. In particular, using photo-transistors instead of microphones allows to have a pulsed light wake-up system operated from a long distance with no propagation delay. Triggering simultaneously a whole environmental wireless sensor network with this solution is possible at a very low power consumption. This solution is reliable even in a dense environment such as a rain forest. This can be used in cooperation with sound detection for trajectography applications [35] in environmental surveys.

\section{ACKNOWLEDGMENT}

The authors would like to thank the Parc National de Port Cros in France for their cooperation in the development and use of our recording systems and acoustic wake-up systems.

The authors would particularly like to thank the Embedded Electronics Technology Platform of Toulon University named SMIoT (Scientific Microsystems for Internet of Things) [37] for its support in the electronic achievement of the project.

\section{REFERENCES}

[1] J. Whitaker, National Audubon Society Field Guide to North American Mammals. National Audubon Society Field Guides, 1996.

[2] S. R. Ganti and Y. Kim, "Implementation of detection and tracking mechanism for small uas," in 2016 International Conference on Unmanned Aircraft Systems (ICUAS), June 2016, pp. 1254-1260.

[3] B. Gestner, J. Tanner, and D. Anderson, "Glass break detector analog front-end using novel classifier circuit," in 2007 IEEE International Symposium on Circuits and Systems, May 2007, pp. 3586-3589.

[4] B. Rumberg, D. W. Graham, V. Kulathumani, and R. Fernandez, "Hibernets: Energy-efficient sensor networks using analog signal processing," IEEE Journal on Emerging and Selected Topics in Circuits and Systems, vol. 1 , no. 3, pp. 321-334, Sept 2011.

[5] R. A. Dobre, V. A. Niţă, A. Ciobanu, C. Negrescu, and D. Stanomir, "Low computational method for siren detection," in 2015 IEEE 21st International Symposium for Design and Technology in Electronic Packaging (SIITME), Oct 2015, pp. 291-295.

[6] V. Gies, V. Barchasz, M. Fourniol, and H. Glotin, "Qualilife-jason advanced sound-light recorder," in 7th International DCLDE (Detection, Classification, Localization, and Density Estimation) Workshop, June 2018.

[7] V. Barchasz, V. Gies, and H. Glotin, "Technical report on high frequency recording systems," Scientific Microsystems for Internet of Things SMIoT, University of Toulon, Tech. Rep., Sept 2017.

[8] E. Alpman, "Overview of low-power wake-up radio design insights," in 2018 IEEE Custom Integrated Circuits Conference (CICC), April 2018, pp. $1-59$.

[9] D. Ghose, F. Y. Li, and V. Pla, "Mac protocols for wake-up radio: Principles, modeling and performance analysis," IEEE Transactions on Industrial Informatics, vol. 14, no. 5, pp. 2294-2306, May 2018.

[10] L. Guntupalli, D. Ghose, F. Y. Li, and M. Gidlund, "Energy efficient consecutive packet transmissions in receiver-initiated wake-up radio enabled wsns," IEEE Sensors Journal, vol. 18, no. 11, pp. 4733-4745, June 2018.

[11] F. Höflinger, G. U. Gamm, J. Albesa, and L. M. Reindl, "Smartphone remote control for home automation applications based on acoustic wake-up receivers," in 2014 IEEE International Instrumentation and Measurement Technology Conference (I2MTC) Proceedings, May 2014, pp. $1580-1583$.

[12] D. H. Goldberg, P. O. Pouliquen, A. G. Andreou, L. Riddle, P. Julian, and R. Rosasco, "A wake-up detector for an acoustic surveillance sensor network: algorithm and vlsi implementation," in Third International Symposium on Information Processing in Sensor Networks, 2004. IPSN 2004, April 2004, pp. 134-141.

[13] H. O. Johansson, "A simple precharged cmos phase frequency detector," IEEE Journal of Solid-State Circuits, vol. 33, no. 2, pp. 295-299, Feb 1998.

[14] A. Sanchez, S. Blanc, P. Yuste, and J. J. Serrano, "Rfid based acoustic wake-up system for underwater sensor networks," in 2011 IEEE Eighth International Conference on Mobile Ad-Hoc and Sensor Systems, Oct 2011, pp. 873-878.

[15] F. Ge and Y. Yan, "Deep neural network based wake-up-word speech recognition with two-stage detection," in 2017 IEEE International Conference on Acoustics, Speech and Signal Processing (ICASSP), March 2017, pp. 2761-2765.

[16] G. Cauwenberghs, A. Andreou, J. West, M. Stanacevic, A. Celik, P. Julian, T. Teixeira, C. Diehl, and L. Riddle, "A miniature low-power intelligent sensor node for persistent acoustic surveillance," vol. 5796, 2005, pp. 5796 - 5796 - 12. [Online]. Available: https://doi.org/10.1117/12.606859 
[17] S. Jeong, Y. Chen, T. Jang, J. Tsai, D. Blaauw, H. S. Kim, and D. Sylvester, "21.6 a 12nw always-on acoustic sensing and object recognition microsystem using frequency-domain feature extraction and svm classification," in 2017 IEEE International Solid-State Circuits Conference (ISSCC), Feb 2017, pp. 362-363.

[18] M. Fourniol, V. Gies, V. Barchasz, E. Kussener, H. Barthelemy, R. Vauche, and H. Glotin, "Low-power wake-up system based on frequency analysis for environmental internet of things," in IEEE International Conference on Mechatronic and Embedded Systems and Applications, July 2018.

[19] R. Kojima, O. Sugiyama, R. Suzuki, K. Nakadai, and C. E. Taylor, "Semi-automatic bird song analysis by spatial-cue-based integration of sound source detection, localization, separation, and identification," in 2016 IEEE/RSJ International Conference on Intelligent Robots and Systems (IROS), Oct 2016, pp. 1287-1292.

[20] K. Nakamura and K. Nakadai, "Robot audition based acoustic event identification using a bayesian model considering spectral and temporal uncertainties," in 2015 IEEE/RSJ International Conference on Intelligent Robots and Systems (IROS), Sept 2015, pp. 4840-4845.

[21] T. Otsuka, K. Ishiguro, H. Sawada, and H. G. Okuno, "Bayesian nonparametrics for microphone array processing," IEEE/ACM Transactions on Audio, Speech, and Language Processing, vol. 22, no. 2, pp. 493504, Feb 2014.

[22] D. Rybach, R. Schlüter, and H. Ney, "Silence is golden: Modeling nonspeech events in wfst-based dynamic network decoders," in 2012 IEEE International Conference on Acoustics, Speech and Signal Processing (ICASSP), March 2012, pp. 4205-4208.

[23] M. L. Chin and J. J. Burred, "Audio event detection based on layered symbolic sequence representations," in 2012 IEEE International Conference on Acoustics, Speech and Signal Processing (ICASSP), March 2012, pp. 1953-1956.

[24] PIC24FJ256GA412/GB412 Family : 16-Bit Flash Microcontrollers with Dual Partition Flash Memory, XLP, LCD, Cryptographic Engine and USB On-The-Go, Microchip Technology Incorporated, 12 2016, rev. 2.0.

[25] Charge Time Measurement Unit (CTMU) and CTMU Operation with Threshold Detect, Microchip Technology Incorporated, 42016.

[26] TPS783xx 500-nA IQ, 150-mA, Ultralow Quiescent Current Low Dropout Linear Regulator, Texas Instruments Incorporated, 112014.

[27] TLV3691 0.9-V to 6.5-V, Nanopower Comparator, Texas Instruments Incorporated, 112015.

[28] TLV7031 and TLV7041 Small Size, nanoPower, Low-Voltage Comparators, Texas Instruments Incorporated, 52018.

[29] CSS555C : Micropower Timer Datasheet, Custom Silicon Solutions, Inc., 52009

[30] CSS555C : Application Circuits - Application Note, Custom Silicon Solutions, Inc., 52009

[31] P. Yedamale and J. Bartling, AN1375 : See What You Can Do with the CTMU, Microchip Technology Incorporated, 22011.

[32] Overview of Charge Time Measurement Unit (CTMU), Microchip Technology Incorporated, 32008.

[33] BCV62 PNP Silicon Double Transistor, Infineon Technologies AG, 7 2011.

[34] REF200 Dual Current Source and Current Sink, Texas Instruments Incorporated, 72015.

[35] M. Fourniol and V. Gies, "Ultra low-power analog wake-up system for environmental iot networks and military smart dust," in submitted to 2018 International Conference on Internet of Things and Intelligence System (IoTaIS), November 2018.

[36] H. Glotin, M. Poupard, F. Monia, J. Ricard, V. Roger, V. Barchasz, V. Gies, and D. Geoffroi, "Analyse temps-réel de masse acoustique pour le suivi d'espèce nuisible à port-cros," Research Report, UTLN CNRS LSIS, Tech. Rep., Dec 2017.

[37] "SMIoT : Scientific microsystems for the internet of things," Available: http://www.smiot.fr, Tech. Rep., 2018. 